\title{
Régie d'entreprise et gouvernance collective comme éléments de la formation et de la répartition du capital au Canada
}

\author{
Marc-André Morency et Jeanne Simard \\ Université du Québec à Chicoutimi
}

\section{Introduction}

Les récents scandales financiers aux États-Unis, en Europe, mais aussi au Canada avec, entre autres, les affaires Nortel, Bre-X, Norbourg, Cinar, ont déclenché à travers le monde une intense activité réglementaire et législative portant sur la régie d'entreprise. Ainsi, aux États-Unis, le Congrès a adopté la Sarbanes-Oxley Act qui édicte de nouvelles mesures pour prévenir et contrer les fraudes ou les malversations financières. Les autorités de la Bourse de New York ont également modifié les conditions d'inscription pour les sociétés cotées et imposé des exigences plus strictes en matière de normes de gestion. La Securities and Exchange Commission a également resserré les conditions afférentes à l'octroi des options d'achat d'actions. Certains des correctifs apportés aux lois américaines ont été rapidement adoptés par les régulateurs canadiens, européens et d'autres pays de l'OCDE. Ces mesures ont pour objectif explicite le maintien de la confiance des investisseurs dans l'intégrité du marché des capitaux. Compte tenu de leur rôle charnière dans la régie d'entreprise, le conseil d'administration et la haute direction ont été le point de mire de ces initiatives de réforme, l'objectif étant de renforcer la fonction de surveillance et d'accroître la responsabilisation des membres du conseil d'administration, investis, en théorie, de l'autorité décisionnelle dans l'entreprise.

Même si ces réformes ont paru constituer un pas dans la bonne direction, plusieurs auteurs estiment qu'elles sont bien loin de protéger vraiment les investisseurs ou les parties prenantes qui, depuis quelques années, ont fait largement les frais des abus commis (congédiements collectifs, comportements prédateurs). Selon eux, les nouvelles dispositions adoptées ne s'attaquent pas à la véritable racine des problèmes observés, en l'occurrence les conflits d'intérêts entre les différents acteurs du marché, soit les bourses, les agences de notation, les banques d'affaires, les analystes, les vérificateurs, les dirigeants, les investisseurs institutionnels, sans oublier les régulateurs eux-mêmes qui bien souvent soutiennent les marchés sans interférer avec leur fonctionnement. $\grave{A}$ titre d'exemple, il suffit d'observer les problèmes associés à la rémunération des dirigeants par voie d'options d'achat d'actions, fruit d'une idéologie critiquée dans ses fondements (la théorie de l'agence et de la perfection des marchés); en ce moment même aux États-Unis, le scandale dévoilé par l'analyse statistique des professeurs Lie et Heron constitue l'illustration parfaite de la dérive de l'appropriation du capital sous ce régime ${ }^{1}$.

On peut avancer qu'en « réformant» simplement le fonctionnement ou la régie des personnes morales et des entreprises, on a voulu conserver le statu quo dans le système économique en fermant les yeux sur bien des dimensions pertinentes à la gouvernance collective, c'est-à-dire aux structures économiques et sociales qui ont fait de la fraude et de la tromperie un problème systémique. En effet, on n'a pas remis en question la déréglementation qui a ouvert la voie à ces scandales. On n'a pas réglé le problème de la répartition du capital construit par le travail collectif. On n'a en rien freiné la hausse vertigineuse des rémunérations captées par les hauts dirigeants. On n'a rien réglé de la détérioration des conditions de vie des travailleurs, des populations et des régions. 
On peut alors comprendre qu'il reste nécessaire de restaurer ou d'instaurer les mécanismes démocratiques, les processus de concertation et de développement, en particulier ceux relatifs à la répartition du capital collectif, entre catégories sociales, entre secteurs, entre régions; tous ces problèmes dépassant, et de loin, les questions de régie interne des organisations et même de régulation du fonctionnement de l'économie ${ }^{2}$.

De façon à mieux situer le recentrage de l'analyse qui s'effectue un peu partout aux dépens du paradigme néolibéral, nous allons rappeler : 1) la distribution des pouvoirs affectant la formation et la distribution du capital au Canada; 2) les scandales ayant suscité une réaction généralisée au pays, ainsi que les mesures adoptées en matière de régie d'entreprise et de gouvernance collective par les législateurs, les organismes de réglementation du commerce des valeurs mobilières et les organismes d'autoréglementation; en 3) nous allons esquisser à grands traits le terrain le plus fondamental où se posent toutes ces questions: les conceptions en conflit, en matière de gouvernance collective et de formation de capital.

\section{Le cadre institutionnel canadien : un aper- çu de notre structure de régie et de gou- vernance collective}

Les provinces se sont vu attribuer par la Constitution canadienne initiale (loi constitutionnelle de 1867) des pouvoirs très larges notamment en matière de propriété, de langue, de droits civils, de sécurité sociale, de santé, d'éducation, d'affaires municipales, de commerce local, de valeurs mobilières, ainsi que la constitution de compagnies pour des fins provinciales. Chaque province a pu adopter sa propre loi régissant les compagnies, accordant aux sociétés par actions le droit d'exercer leurs objets et entreprises, et ce, dans les limites de leur juridiction. Ces personnes morales peuvent opérer hors du territoire provincial, avec la permission des autres provinces ou territoires. Chaque province a l'autorité exclusive d'encadrer le marché des valeurs mobilières sur son territoire. En 1955, le Québec mettait sur pieds la Commission des valeurs mobilières (intégrée depuis 2004 à la nouvelle Autorité des marchés financiers). L'Autorité a pour mission de protéger les épargnants et d'assurer l'encadrement des marchés des valeurs mobilières, de veiller à ce que les émetteurs et les autres intervenants du secteur financier (courtiers, conseillers en valeurs, certains organismes d'autoréglementation dont la Bourse de Montréal) se conforment aux obligations qui leur sont applicables en vertu de la Loi sur les valeurs mobilières du Québec. Cette Autorité dispose de pouvoirs discrétionnaires lui permettant d'adopter des normes de portée générale, des règlements, des instructions, enfin de rendre des décisions sur des cas particuliers. Cette Autorité travaille également de concert avec les autres autorités provinciales ou canadiennes.

Par contraste, la loi constitutionnelle canadienne ne prévoit aucune disposition expresse correspondante permettant de fonder la compétence fédérale en droit des compagnies et en droit des valeurs mobilières. La jurisprudence et la doctrine ont néanmoins reconnu au Parlement fédéral le pouvoir de régir la constitution de sociétés canadiennes en invoquant principalement un dispositif de la constitution qui confère au Parlement fédéral une compétence résiduelle, c'est-à-dire le pouvoir d'adopter des lois pour la paix, l'ordre et le bon gouvernement dans les matières qui n'ont pas été accordées de manière exclusive aux provinces. Ainsi, le fédéral a pu adopter en 1974 la Loi canadienne sur les sociétés par actions. En ce qui concerne le marché des valeurs mobilières, le gouvernement fédéral n'en a pas la responsabilité constitutionnelle. Toutefois, les autorités des provinces et des territoires ont pu s'associer et créer un forum, le regroupement des Autorités canadiennes en valeurs mobilières, permettant ainsi aux 13 organismes la réglementation du commerce des valeurs mobilières des provinces (dont le Québec) et des territoires, de coordonner et d'harmoniser la réglementation des marchés financiers d'un bout à l'autre du pays. Tout récemment, le gouvernement ontarien indiquait son intérêt de voir s'établir une commission nationale des valeurs mobilières. Le gouvernement fédéral a alors fait remarquer qu'une telle autorité devrait résulter de la volonté exprimée des provinces, non d'une initiative fédérale ${ }^{3}$.

Chaque province a l'autorité exclusive

d'encadrer le marché des valeurs mobilières sur son territoire. 


\section{Quelques scandales financiers : impacts et conséquences juridiques}

Wade Rowland, dans son ouvrage intitulé La soif des entreprises ${ }^{4}$, a bien établi la nécessité d'opérer la distinction entre deux types de comportements scandaleux : il y a d'une part les crimes commis à l'encontre de la personne morale, de l'entreprise et des actionnaires; c'est ce que l'on tente habituellement de prévenir ou de dénoncer dans la presse économique. Mais il y a une dimension occultée dont on parle beaucoup moins : ce sont les crimes commis par les personnes morales à l'encontre des sociétés, c'est-à-dire du contexte social.

\section{Les crimes contre la personne morale, l'entreprise et les actionnaires}

Le contexte global favorisait depuis 1970 une relative passivité des autorités chargées de réglementer le marché des valeurs mobilières. L'ampleur des scandales chez nos voisins américains et la vigueur des initiatives y répondant ont créé une énorme pression pour que les autorités gouvernementales canadiennes interviennent rapidement afin d'endiguer les risques de réalisation chez nous de tels événements. Il faut dire qu'en matière de scandales financiers (tels l'usage d'informations privilégiées, la manipulation du marché, la corruption, le détournement de fonds, la falsification de l'image de l'entreprise), le Canada et le Québec n'ont malheureusement rien à envier aux États-Unis. Qu'on pense au Fond de placement Norbourg, dans lequel 9200 investisseurs ont été floués de 130 millions de dollars par son principal dirigeant. Rappelons également le scandale Cinar (2000), dans lequel les fondateurs d'une société de production télévisuelle québécoise ont détourné des millions de dollars en crédits d'impôt par l'utilisation de prête-noms. Les fondateurs auraient fait disparaître 123 millions de dollars des coffres de la société pour les «déplacer » à l'étranger, sans avoir préalablement obtenu l'autorisation du conseil d'administration. Enfin, le scandale des scandales est celui de Nortel, le numéro un mondial de la transmission de données par fibre optique. La dégringolade boursière de la plus importante entreprise canadienne a occasionné des pertes boursières évaluées à quelques 400 milliards de dollars, soit de 4 à 5 fois en importance les pertes encourues dans l'affaire Enron. À la fin des années
90, une série d'acquisitions surévaluées ont miné sa rentabilité. Ses dirigeants ont troqué des pratiques comptables respectueuses des règles de l'art pour des «formules créatives », des falsifications qui ont permis à l'entreprise d'annoncer des bénéfices nets d'exploitation de plusieurs milliards, alors que dans les faits, elle aurait dû afficher d'importantes pertes. Le pire dans tout cela est que des investisseurs avisés, des observateurs réputés, des analystes, des maisons de courtage et même le conseil d'administration de l'entreprise, n'y ont vu que du feu, et alors même que des dirigeants fuyaient ou étaient chassés tout en bénéficiant de fabuleux parachutes dorés, des options d'achat exercées, etc. Encore à ce jour, il n'y a pas eu d'enquête publique mais certains dirigeants font maintenant l'objet de poursuites. Rappelons que 60000 personnes y ont perdu leur emploi en l'espace de deux ans ${ }^{5}$. En 2002, Nortel a opté pour un règlement hors cour avec un collectif qui la poursuivait.

À la suite de l'affaire Nortel, les bourses canadiennes (qui étaient jusqu'alors considérées parmi les plus performantes au monde) sont devenues très inquiètes en raison de la perte de confiance des investisseurs dans le marché. Elles ont formé un comité de révision des règles de régie des entreprises canadiennes. Étonnamment, ce comité a été présidé par un membre du conseil d'administation de Nortel, Mme Guylaine Saucier. Le rapport Saucier, déposé en novembre 2001, proposait 15 recommandations destinées à améliorer la responsabilité générale de gérance du patrimoine des sociétés, préconisant une approche purement incitative, et nous prévenant des inconvénients d'adopter des approches contraignantes ! Le rapport affirme que...

si certains aspects de la «gouvernance » peuvent se prêter à la réglementation, la divulgation d'information constitue une méthode bien meilleure de réglementation du comportement, lorsqu'on vise à susciter l'émergence d'une saine culture de gouvernance ${ }^{6}$.

À la suite de l'affaire Nortel, les bourses canadiennes (qui étaient jusqu'alors considérées parmi les plus performantes au monde) sont devenues très inquiètes en raison de la perte de confiance des investisseurs dans le marché. 
Selon cette perspective par ailleurs souvent évoquée, mieux vaut développer le sens de l'éthique, la conscience professionnelle que, par interdits, conduire à une situation où l'on s'ingénue à faire tout ce qui n'est pas interdit, en vertu du dicton: «Si ce n'est pas interdit, c'est donc que c'est permis !». Ainsi, le rapport Saucier restait muet quant aux obligations des conseils d'administration et leurs responsabilités vis-à-vis les intérêts des parties prenantes, c'est-à-dire les divers groupes directement touchés par l'activité de l'entreprise.

En réponse à ces grands scandales qui ont surgi avec l'éclatement de la bulle spéculative de la nouvelle économie, les États-Unis adoptaient, en 2002, la Loi Sarbanes-Oxley, qui apporte des changements radicaux à la régie d'entreprise et à l'obligation de divulguer des sociétés cotées en bourse. Par conséquent, pour assurer une certaine compatibilité entre les deux pays et la compétitivité du Canada, les régulateurs canadiens ont dû adopter des mesures analogues. La réponse du Canada a toutefois été déterminée par les conditions uniques de son marché intérieur où le contrôle effectif de plusieurs grandes sociétés se retrouve entre les mains de quelques familles richissimes et fort influentes. Selon Gilles Paquet, « le Canada aurait moins souffert des difficultés à contrôler les gestionnaires que les entreprises américaines dont l'actionnariat est plus éclaté $\gg \mathrm{m}^{7}$. D'ailleurs, les investisseurs institutionnels (par exemple la Caisse de dépôt de placement, le Fonds de retraite des enseignants de l'Ontario) et les grandes banques, qui sont propriétaires de la majorité (environ $58 \%$ ) de tous les titres canadiens en circulation, ne voient pas toujours d'un très bon œil l'intervention musclée du législateur en matière de régie d'entreprise, désireux qu'ils sont d'afficher une bonne performance périodique à leurs épargnants ou retraités.

Voyons donc certaines des mesures adoptées (ou présentement en chantier) par les organismes de réglementation du commerce des valeurs mobilières, par les gouvernements fédéral et provinciaux et par les organismes d'autoréglementation ${ }^{8}$.

\section{Les Autorités canadiennes en valeurs mobilières}

Le regroupement des Autorités canadiennes en valeurs mobilières, après de vastes consultations fai- sant référence pour la plupart aux dispositions de la Loi Sarbanes-Oxley, a adopté une série de règlements et de directives visant à augmenter la qualité et l'intégrité de la divulgation d'information en matière de régie d'entreprise. Certains de ces règlements et directives sont obligatoires, d'autres ne le sont pas. Sont entrés en vigueur au milieu de 2005 :

le règlement sur le comité de vérification (52-110) lequel exige que tout émetteur ait un comité chargé d'évaluer objectivement la justesse des pratiques des dirigeants en matière de comptabilité et de divulgation d'information. Ce comité doit être composé d'au moins trois administrateurs indépendants de l'émetteur et posséder des compétences financières. Il doit surveiller les travaux du vérificateur externe et approuver au préalable tous les services non liés à la certification des états financiers;

le règlement sur l'attestation de l'information présenté dans les documents annuels et intermédiaires des sociétés (52-109), qui impose au chef de direction et au chef des finances l'obligation, sous peine de poursuites personnelles, de fournir des attestations concernant l'information présentée dans les documents annuels et intermédiaires de l'émetteur;

le règlement sur la surveillance des vérificateurscomptables (52-108), qui vise à renforcer la confiance du public dans l'intégrité de l'information financière des sociétés en favorisant une vérification indépendante de haute qualité. En vertu du règlement, la société cotée en bourse qui dépose des états financiers accompagnés d'un rapport de vérification doit faire établir ce rapport par un cabinet d'expertscomptables participant au programme d'un nouvel organisme, le Conseil canadien de reddition des comptes (CCRC). En effet, les Autorités canadiennes en valeurs mobilières, le surintendant des institutions financières du Canada et l'Institut canadien des comptables agréés ont créé cet organisme de surveillance et de contrôle des vérificateurs de sociétés publiques. Cet organisme privé a le pouvoir de formuler des règles en matière d'indépendance et de contrôle de la qualité pour les cabinets et d'effectuer une inspection professionnelle des vérificateurs des sociétés cotées.

Par contre, le règlement sur l'information concernant les pratiques en matière de gouvernance (58- 
101) ne propose pas une liste de pratiques exemplaires des entreprises canadiennes; il établit plutôt des obligations de divulgation précises sur les pratiques de régie d'entreprise des émetteurs, entre autres concernant : a) l'indépendance des administrateurs; b) les mesures prises par le conseil d'administration pour orienter les nouveaux administrateurs dans leur fonction et pour assurer la formation continue (s'il en est); c) l'adoption d'un code écrit à l'intention des dirigeants et des salariés; d) les mesures prises pour assurer une procédure objective de détermination de la rémunération des administrateurs et des dirigeants, etc.

Certaines Autorités canadiennes en valeurs mobilières (à l'exception de la Colombie-Britannique, de l'Alberta et du Québec) ont voulu aller plus loin encore en adoptant une instruction générale (58201) qui énonce plus spécifiquement des pratiques exemplaires de régie d'entreprise, (composition du conseil d'administration, réunions des administrateurs indépendants, mandat du conseil d'administration, contenu et gestion d'un code de conduite et d'éthique, sélection des candidats au conseil d'administration, composition et le fonctionnement du comité de rémunération des dirigeants). Ces lignes de conduite n'étant pas obligatoires, tout ce que doivent faire les émetteurs, c'est divulguer leurs propres pratiques de régie d'entreprise en les comparant à ces pratiques exemplaires et, le cas échéant, justifier toute dérogation à celles-ci. Aux participants sur le marché de déterminer si l'entreprise réalise ou dépasse les attentes à cet égard.

\section{La loi prévoit des mesures de protection pour les employés qui dénonceraient les pratiques douteuses de leur employeur.}

\section{Le gouvernement fédéral}

Le 12 février 2004, le gouvernement fédéral modifiait à nouveau le Code criminel, afin de renforcer cette fois-ci les mesures d'investigation, de poursuite et de dissuasion relatives à la fraude sur les marchés financiers. Il s'agissait de rendre plus incisive une loi longtemps considérée faible et inefficace en ce qui concerne la poursuite des contrevenants en matière de fraude. Spécifiquement, le législateur a créé une nouvelle infraction pénale visant les délits d'initiés. La loi prévoit des mesures de protection pour les employés qui dénonceraient les pratiques douteuses de leur employeur. Elle augmente les peines maximales applicables aux délits de fraude (les faisant passer de 10 à 14 ans) et dresse une liste de circonstances aggravantes pour aider les tribunaux à déterminer les sentences. Les peines en vertu des lois américaines sont en fait deux fois plus sévères. Mais au Canada, en raison de la rareté des peines infligées, du faible nombre de poursuites, de l'extrême indulgence des tribunaux, cette mise à jour constitue un signal important à l'endroit des gens d'affaires.

Dans un livre blanc de consultation, Industrie Canada a publié en mai 2004 des propositions de modification à la Loi canadienne sur les sociétés par actions visant à renforcer la régie d'entreprise des sociétés constituées en vertu de cette loi. Certaines propositions visant à la codification des meilleures pratiques ont été visiblement inspirées par celles proposées par les Autorités canadiennes en valeurs mobilières et pourraient devenir obligatoires pour les sociétés constituées au niveau fédéral. À notre connaissance, cette consultation est toujours en cours, le processus de révision quinquennal n'étant pas terminé.

\section{Le gouvernement québécois}

Au Québec, l'Autorité des marchés financiers est née le 1er février 2004 de la fusion de la Commission des valeurs mobilières du Québec, du Bureau des services financiers, de la Régie de l'assurancedépôts, du Fonds d'indemnisation des services financiers et de l'Inspecteur général des institutions financières. L'objectif de sa création est notamment de faciliter la vie des consommateurs de ces services en leur offrant un guichet unique pour le traitement des plaintes et la réception des documents issus des émetteurs. Il ne semble pas y avoir de modification de la Loi sur les compagnies québécoises en préparation.

\section{Les ordres professionnels}

Sur certains aspects touchant la vérification comptable et les services d'analyse-conseil, l'Institut canadien des comptables agréés a adopté en 2003 des normes d'indépendance semblables à celles qui ont été adoptées aux États-Unis, et elles ont déjà été 
intégrées dans certains codes de déontologie des ordres provinciaux de comptables agréés.

Incidemment, il convient de souligner l'insertion dans son code de déontologie, par les Administrateurs agréés du Québec, d'un corpus de normes de saine gestion. Cette insertion fait en sorte que l'obligation de comportement éthique en matière de conséquences de pratiques «managériales » pour la société civile et l'organisation, se voit offrir un ensemble intégré de balises permettant de jauger les meilleures approches en matière de gestion ${ }^{9}$.

\section{Les activités d'autorégulation}

Plusieurs grands investisseurs institutionnels canadiens ont entrepris de créer la Canadian Coalition for Good Governance, afin de mieux faire connaître leurs attentes en matière de régie d'entreprise. Cette coalition regroupe des organisations comme l'Ontario Teachers Pension Plan Board, le Canada Pension Plan Investment Board et, notamment, Jarislowsky Fraser Ltd. Ce sont des joueurs de premier niveau, qui contrôlent $58 \%$ du marché, soit 425 milliards \$; ils ont publié en 2003 un ensemble de lignes directrices à l'intention des compagnies dans lesquelles ils investissent. Cette activité n'est pas sans rappeler qu'au Royaume-Uni, les investisseurs institutionnels ont aussi été mobilisés par les autorités gouvernementales afin de promouvoir des réformes au sein des entreprises bénéficiant de leurs investissements.

Les crimes commis par les personnes morales contre la société

Au-delà des problèmes relatifs aux aspects internes des personnes morales, plusieurs cas de crimes commis par les personnes morales contre la société ont aussi été reconnus et sanctionnés. Le cas de la Union Carbide Corporation en est un exemple. Cette firme opérait à Bhopal, en Inde, une usine de produits chimiques à proximité d'une importante communauté humaine. Le 3 décembre 1984, l'explosion d'une de ses usines de pesticide a dégagé 40 tonnes d'isocyanate de méthyle dans l'atmosphère de la ville, tuant entre 16000 et 30000 personnes. La composition du gaz ne fut pas dévoilée pour des raisons de secret industriel. Plus près de nous, Vêtements de sport Gildan, le plus gros fabricant de tshirts au Québec a vu son statut au sein du Fair La- bor Association (un organisme de certification sociale) sérieusement remis en question lorsqu'il a dû, en 2004, faire face à deux rapports incriminants provenant d'organismes indépendants, sur les conditions de travail de misère prévalant dans son usine au Honduras. De même, plusieurs compagnies canadiennes exploitant des mines en Afrique font l'objet de critiques virulentes en ce qui concerne le peu de cas qu'elles font de l'environnement et des conditions de travail de leurs employés.

\section{Il convient de souligner l'insertion dans son code de déontologie, par les Administra- teurs agréés du Québec, d'un corpus de normes de saine gestion.}

Sur le plan collectif, avec la multiplication des regards critiques portés sur ces aspects troubles et criminels de la mondialisation, on a vu se développer une prise de conscience voulant que même si la création de valeur est la première raison d'être de la personne morale, celle-ci doit aussi prendre en compte les groupes d'intérêts touchés par ses opérations et faire preuve de conscience sociale, bref agir comme personne morale dans l'intérêt du public, avec le souci de la protection du public. Les nombreuses manifestations au Canada en faveur des droits de la personne et de l'environnement illustrent le fait que les sociétés sont appelées de plus en plus à se montrer bonnes citoyennes si elles ne veulent pas faire l'objet de boycott, perdre leur réputation et la confiance des investisseurs. En 2001, la Commission sur la démocratie canadienne et la responsabilisation des entreprises a dépassé la question de la régie d'entreprise pour poser de façon plus globale la problématique de la gouvernance collective. La Commission a montré qu'il s'agit bel et bien de renoncer précisément à une position idyllique fondée sur le bon vouloir, et de déterminer un plancher, un "level playing field » minimum pour tous, au Canada, et même dans les opérations à l'étranger ${ }^{10}$.

En 2002, le gouvernement québécois s'est lui aussi joint au débat en tenant une commission parlementaire pour déterminer s'il doit légiférer en matière de responsabilité sociale. La question soulevée était la suivante : "Compte tenu de la place occupée par la responsabilité sociale des entreprises et l'investissement responsable, quel rôle le gouver- 
nement est-il appelé à jouer dans ses interventions auprès du secteur privé et dans ses propres activités ? \' C'était rappeler que l'État aussi investit, qu'il répartit une part importante du capital commun, bref qu'il est un joueur économique avec qui compter. Cette commission n'a pas reçu l'attention qu'elle méritait en raison d'un changement de gouvernement.

Par contre, même s'il n'y a pas eu d'initiative globale comme en France où le législateur oblige les entreprises à remettre annuellement un bilan social et environnemental, le Canada a adopté, en 1999, la Loi sur la corruption des agents publics étrangers. Il sera plus difficile maintenant de justifier des surcoûts afférents à la rémunération d'agents publics étrangers dans l'exercice de leurs devoirs. On tolère seulement les coûts raisonnables afférents au traitement accéléré de processus bureaucratiques. Cette loi est très limitée dans sa portée, mais elle a valeur symbolique et critique dans l'état actuel des pratiques concurrentielles dans la guerre économique en cours.

\section{Nous estimons que les réformes en matière de régie d'entreprise et de gouvernance collective demeurent à bien des égards in- suffisantes.}

Conséquences des engagements du Canada (Statut de Rome de la Cour pénale internationale), depuis 2000, la Loi sur les crimes contre l'humanité et les crimes de guerre intègre de nouvelles infractions au Code criminel. Les autorités canadiennes peuvent intenter des poursuites contre les entreprises canadiennes ainsi que le personnel de direction impliqués dans des opérations criminelles effectuées dans des zones troubles de la scène planétaire. Il convient de souligner que la responsabilité pénale touche aussi bien la personne morale que les personnes physiques impliquées. La guerre économique, qui peut à la limite se révéler une politique d'expansion effectuée "par d'autres moyens », trouve ainsi son pendant criminel par le truchement d'une loi à portée extraterritoriale.

Enfin, en 2006, le gouvernement du Québec promulguait la Loi sur le développement durable dont le titre 1 se lit Gouvernance fondée sur le développement durable. Son objet est d'instaurer un nou- veau cadre de gestion au sein de l'Administration. Comme l'Administration publique et les administrations privées sont appelées de plus en plus à agir de concert, l'impact d'une telle loi risque d'être considérable. Elle mérite d'être suivie de près.

\section{Pour une réforme de la régie et de la gou- vernance collective}

Nous estimons que les réformes en matière de régie d'entreprise et de gouvernance collective demeurent, à bien des égards, insuffisantes. Par leur réponse aux scandales financiers, les autorités canadiennes ont généralement imité, sur le fond, la perspective américaine touchant aux rapports entre dirigeants, administrateurs et actionnaires. Presque tous les efforts sont consacrés à éviter que les dirigeants n'utilisent leurs pouvoirs à mauvais escient pour capturer les profits. C'est se rabattre sur une conception réductrice et erronée de la société par actions qui en fait la "propriété » des actionnaires, devant être administrée en fonction de leurs seuls intérêts. L'analyse économique tente de justifier une telle conception en soulignant les risques non contractualisables que les actionnaires supportent dans le cadre de l'activité entrepreneuriale.

Pourtant, en 2004, la Cour suprême du Canada dans l'affaire People c. Wise a critiqué cette façon étroite de concevoir le devoir de loyauté des administrateurs et des dirigeants. Dans son jugement, la cour rappelle que l'article 122 de la Loi canadienne sur les sociétés par actions mentionne très clairement « que les administrateurs et les dirigeants doivent, dans l'exercice de leurs fonctions, agir avec intégrité et bonne foi au mieux des intérêts de la société (par actions) » entendu ici comme la personne morale et comme entreprise, c'est-à-dire une organisation durable dans le temps. On ne doit jamais perdre de vue qu'une entreprise est une communauté productive autonome où s'investissent plusieurs intervenants ou partenaires. Par conséquent, le conseil d'administration, lorsqu'il examine l'ensemble des circonstances dans un cas donné, doit tenir compte notamment des intérêts des actionnaires, mais aussi des employés, des fournisseurs, des créanciers, des consommateurs, des gouvernements et des collectivités environnantes. Les actionnaires s'investissent financièrement, mais ces autres parties prenantes comme les salariés, les managers par exemple, en- 
gagent leurs ressources, mais pas seulement sous une forme financière. Cet «investissement» du capital humain et social est tout aussi important pour la pérennité de l'entreprise à long terme. Selon Aglietta et Rebérioux,

La dimension partenariale de la firme doit s'affirmer: elle se donne à voir comme la réunion des compétences stratégiques, cognitives et financières qui assurent le développement et la compétitivité de l'entité ainsi constituée ${ }^{11}$.

Or, avec les récentes modifications apportées à la régie d'entreprise au Canada, force est de constater qu'il subsiste plus qu'une simple méconnaissance ou interprétation erronée de la Loi canadienne sur les sociétés par actions : il existe en fait un conflit de perspectives, un enjeu de classes pour la gouverne du pays. Soit on s'assure que la régie d'entreprise préserve l'intérêt de la société par actions et les progrès sociaux qu'elle procure à la nation, soit on accorde de fait aux actionnaires et aux financiers une possibilité illimitée de prélèvement du capital financier et de son déplacement vers des intérêts globaux indéterminés. C'est ce passage d'un capitalisme national vers un capitalisme délocalisé et dénué de toutes considérations éthiques, déontologiques, ou d'ancrage dans la communauté territoriale, que Weber observait déjà en formation, il y a 100 ans, aux Etats-Unis ${ }^{12}$. C'est cette dernière conception qui se manifeste lorsque nous empruntons aveuglement à nos voisins leurs timides réformes et leurs mouvements vers la déréglementation qui se fondent sur une exigence de rentabilité financière immédiate. La production de valeur de la firme pour le marché des valeurs mobilières s'impose alors comme le seul critère de réussite d'une entreprise, bref comme une détermination ex ante, qui enlève toute perspective stratégique globale à la régie d'entreprise.

On comprend alors les préoccupations manifestées lors du périple pancanadien de la Commission sur la démocratie canadienne et la responsabilisation des entreprises, dont le rapport visait à renforcer la $L o i$ canadienne sur les sociétés par actions, mais aussi les lois qui protègent les salariés et les collectivités nationales contre les restructurations massives et le poids des baisses continuelles d'impôt en faveur du milieu des affaires. En effet, seuls les États peuvent, s'ils n'abdiquent pas leur responsabilité propre, indiquer aux sociétés par actions, ainsi qu'à l'ensemble des acteurs sociaux, le socle commun des observances et règles propres à assurer le bienêtre commun sans lequel la vie sociale ne peut exister. L'État peut le faire, pour la collectivité, à la condition de conserver évidemment son pouvoir effectif et financier de contrainte et de sanction. Ce que la mondialisation néolibérale propagée par les grandes sociétés par actions cherche sans relâche à éroder. On ne peut manquer d'observer que les grands opérateurs privés procèdent actuellement à une intense recherche visant la fusion ou l'achat d'entreprises cotées, afin de les privatiser, les rendant ainsi moins vulnérables à l'attention renouvelée des régulateurs. La SEC vient tout de même de déclencher une vaste enquête sur une vague de mouvements boursiers précédant les opérations publiques de rachat et fusion. On soupçonne que les délits d'initiés sont toujours une règle implicite de la gouvernance des entreprises...

Autre problème non-résolu : la délibération démocratique fait présentement défaut à l'échelon planétaire, à l'échelle des pays et au sein des organisations. C'est là le plus important travail à effectuer pour notre génération : cesser de croire à la magie intrinsèque des automatismes du marché et instaurer la vie démocratique à ces divers échelons. Afin de renouer avec le progrès social, il paraît indispensable de s'interroger sur la légitimité des règles de régie d'entreprise telles qu'elles sont présentement définies, car elles ne donnent que peu de place à une vraie délibération entre les différentes parties prenantes d'une entreprise. C'est l'ensemble de ces parties qui devra à l'avenir définir les intérêts sociaux des entreprises et par conséquent leur efficacité économique et sociale.

\section{Il paraît indispensable de s'interroger sur la légitimité des règles de régie d'entreprise telles qu'elles sont présentement définies.}

Cet effort vers une véritable démocratie économique ne se fera pas sans heurts. L'État-nation, même s'il veut intervenir, doit faire face à des contraintes majeures issues de l'extérieur, dans un cadre que l'on décrit actuellement comme une "guerre économique » à l'échelon global. Cette guerre qui a été 
l'objet de nombreuses réflexions depuis des décennies en France et ailleurs met à jour les jeux d'hégémonie que pratiquent les grandes puissances et leurs entreprises à vocation globale; c'est notamment le cas de la Chine, de la Communauté européenne et des États-Unis. Le level playing field construit par les petits et moyens États nationaux est sapé par les interventions d'oligopoles mondiaux (certaines entreprises multinationales, transnationales qui sont aussi riches, parfois plus riches que les États) qui mettent en place les règles plus ou moins hétéroclites du "droit souple », c'est-à-dire des pratiques de gouvernance éclatées et particularisées. Un hyperféodalisme mondial remodèle les territoires en fonction des seules perspectives des forces dominantes. Plusieurs observateurs soulignent notamment que la solution de ces problèmes ne saurait résulter de l'accumulation de compromis locaux engendrés dans la foulée de ces définitions particulières de l'intérêt social.

Ces analyses critiques contribuent à nous libérer des représentations fondées sur des perspectives trop abstraites ou fondées sur l'individualisme érigé en doctrine. Ainsi, le marché nous apparaît comme un vrai jeu complexe d'acteurs ou de forces sociales inégales à l'échelle d'un système mondial émergent et non comme une figure abstraite du langage microéconomique. On comprend alors que c'est seulement par un réel développement de la conscience sociale que la régie d'entreprise pourra servir non seulement les parties prenantes de l'entreprise dans son milieu immédiat, mais aussi les objectifs sociétaux du développement durable et les autres principes de survie des sociétés historiques. Les effets territoriaux des solutions retenues pourront alors se trouver examinés dans le cadre de gouvernance qui leur convient, chaque organisation s'orientant au produit optimum à livrer dans le contexte où elle se trouve inscrite.

\section{Notes et références}

1 Heron, R. A., E. Lie (2006). "Does Backdating Explain the Stock Price Pattern Around Executive Stock Option Grants ?" Journal of Financial Economics. Ce document peut être consulté à l'adresse suivante : http://home.kelley.iupui.edu/rheron/grantsfinal. pdf.

2 Pour bien comprendre les nouvelles règles affectant la régie d'entreprise, on ne peut se dispenser de situer ces microchangements institutionnels au sein d'une pro- blématique beaucoup plus large qui est celle de la gouvernance collective. Il est désolant de constater qu'au cours des dernières années, il y a eu un glissement sémantique (inspiré par le mouvement de la corporate governance) assimilant la régie d'entreprise à la notion beaucoup plus large de gouvernance. La gouvernance touche les orientations d'une communauté humaine à travers ses différentes institutions publiques et privées; il s'agit de la détermination des objectifs fondamentaux relatifs à l'existence de cette communauté, à son développement durable, à l'attribution des responsabilités en matière décisionnelle, au rôle de la démocratie et à son évolution. C'est ainsi que la formation de capital dans un pays doit être pensée, non comme le fait du libre jeu d'un marché autonome supposé parfait et naturellement efficient, mais bien plutôt comme la résultante d'une multitude de décisions prises par des acteurs publics et privés, individus, corporations, communautés, enfin par tout un réseau d'acteurs de la société civile. La formation de capital traduit en fait l'évolution d'ensemble d'une société donnée. C'est pourquoi le concept de gouvernance doit être conceptuellement réservé à ce niveau de la réalité et non réduite à analyser les modus operandi des organisations. Toute confusion de cette nature trahit l'influence de l'idéologie sur ses propagandistes.

3 Boisvert, M. (2006), « Le mirage du siège social », $L a$ Presse Affaires, vendredi, 20 janvier 2006, p. 7.

4 Rowland, W. (2006), La soif des entreprises, Montréal, Éditions Hurtubise HMH ltée.

5 Zone libre enquêtes, Radio-Canada, Le scandale financier Nortel, http://www.src.info/actualite/v2/ZoneLibre/ (le 27 janvier 2006).

6 Comité mixte sur la gouvernance d'entreprise (2001). « Au-delà de la conformité, la gouvernance... », (Présidé par Mme Guylaine Saucier).

7 Paquet, G. (2005). Gouvernance. Une invitation à la subversion. Montréal, Liber, p. 203.

8 Pour plus de détails, on lira : Rousseau, S. (2004). « Le rôle des tribunaux et du conseil d'administration dans la gouvernance des sociétés ouvertes : réflexions sur la règle du jugement d'affaire ", Les Cahiers de Droit, vol. 45, nº 3, p. 469-540; Martel, P. (2004). « Après Enron, Worldcom et les autres : veut-on vraiment protéger les investisseurs? \, dans Valeurs mobilières et protection du public: le point de vue de l'investisseur, Montréal, p. 67-105; De Serres, A. (2004), « Quel rôle pour le législateur en matière de gouvernance d'entreprises ? Un aperçu des modes d'intervention préconisés aux États-Unis, au Royaume-Uni, en France, au Canada et au Québec », dans Valeurs mobilières et protection du public : le point de vue de l'investisseur. Actes du Colloque UQAM/CVMQ. Montréal: Wilson \& Lafleur/Martel 21-48. 
9 Simard, J., Morency, M.-A. (2006). Commentaires sur le Code de déontologie des administrateurs agréés du Québec, Cahier de recherche, Chaire de responsabilité sociale et de développement durable, Université du Québec à Montréal.

Commission sur la démocratie canadienne et la responsabilisation des entreprises (2002), Rapport final :

une nouvelle équation; les profits et les responsabilités des entreprises à l'aube du 21 siècle. Ce document peut être consulté à l'adresse suivante : http://www.corporate-accountability.ca.

11 Aglietta, M. et Rebérioux, A. (2004), Dérives du capitalisme financier, Paris, Albin Michel, p. 71

12 Max Weber (2002), « Les sectes protestantes et l'esprit du capitalisme », L'éthique protestante et l'esprit du capitalisme, Paris, Flammarion, traduction de Isabelle Kalinowski, $3^{\text {e }}$ éd., p. 305-315. 